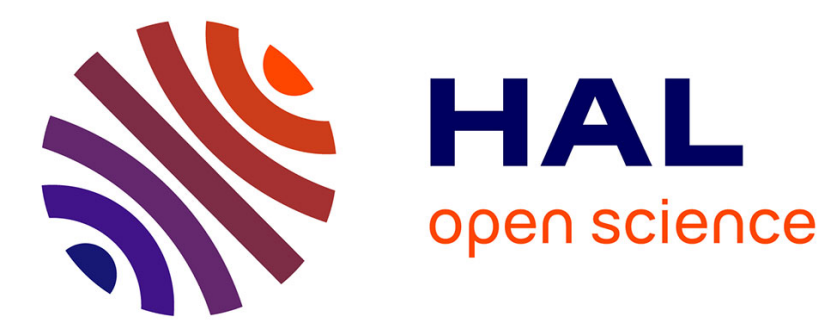

\title{
Speak on time! Effects of a musical rhythmic training on children with hearing loss
}

Céline Hidalgo, Simone Falk, Daniele Schön

\section{To cite this version:}

Céline Hidalgo, Simone Falk, Daniele Schön. Speak on time! Effects of a musical rhythmic training on children with hearing loss. Hearing Research, 2017, 10.1016/j.heares.2017.05.006 . hal-01577772

HAL Id: hal-01577772

https://hal.science/hal-01577772

Submitted on 28 Aug 2017

HAL is a multi-disciplinary open access archive for the deposit and dissemination of scientific research documents, whether they are published or not. The documents may come from teaching and research institutions in France or abroad, or from public or private research centers.
L'archive ouverte pluridisciplinaire HAL, est destinée au dépôt et à la diffusion de documents scientifiques de niveau recherche, publiés ou non, émanant des établissements d'enseignement et de recherche français ou étrangers, des laboratoires publics ou privés. 
1

2 Speak on time! Effects of a musical rhythmic training on children with hearing loss 3

4

5

6

19 Corresponding author: Céline Hidalgo, celine.hidalgo@univ-amu.fr, Laboratoire Parole et Langage, UMR 7309, CNRS, Aix-Marseille University, 5 Avenue Pasteur, 13100 Aix-en-Provence,

\section{Céline Hidalgo ${ }^{1,4}$, Simone Falk ${ }^{1,2,3}$, Daniele Schön ${ }^{4}$}

${ }^{1}$ Aix Marseille Univ, CNRS, LPL, Aix-en Provence, France

${ }^{2}$ Clinical \& German Linguistics, Ludwig-Maximilians-University Munich, Germany

${ }^{3}$ Laboratoire Phonétique et Phonologie, UMR 7018, CNRS, Université Sorbonne Nouvelle Paris-3, France

${ }^{4}$ Aix Marseille Univ, INSERM, INS, Inst Neurosci Syst, Marseille, France 
Temporal accommodation in children with HL

\section{Abstract}

26 This study investigates temporal adaptation in speech interaction in children with normal hearing

27 and in children with cochlear implants (CIs) and/or hearing aids (HAs). We also address the ques-

28 tion of whether musical rhythmic training can improve these skills in children with hearing loss

29 (HL). Children named pictures presented on the screen in alternation with a virtual partner. Alterna-

30 tion rate (fast or slow) and the temporal predictability (match vs mismatch of stress occurrences)

31 were manipulated. One group of children with normal hearing (NH) and one with HL were tested.

32 The latter group was tested twice: once after 30 minutes of speech therapy and once after 30

33 minutes of musical rhythmic training. Both groups of children (NH and with HL) can adjust their

34 speech production to the rate of alternation of the virtual partner. Moreover, while children with

35 normal hearing benefit from the temporal regularity of stress occurrences, children with HL become

36 sensitive to this manipulation only after rhythmic training. Rhythmic training may help children

37 with HL to structure the temporal flow of their verbal interactions.

\section{$38 \underline{\text { Keywords }}$}

39 Hearing loss; Children; Temporal accommodation; Speech production; Interaction; Rhythmic train-

40 ing.

$41 \quad \underline{\text { Abbreviations }}$

$42 \mathrm{NH}$, normal hearing; HL, hearing loss; P-center, perceptual center; IWI, inter-word-interval; ITI, 43 inter-turn-interval; CI, cochlear implant; HA, hearing aid. 
Temporal accommodation in children with HL

\section{1. Introduction}

45 Conversation requires that speakers accommodate to the verbal behavior of their conversational

46 partner. This accommodation can alter the content (e.g. semantic system, Garrod \& Anderson,

47 1987), the form of the exchange (e.g. intensity, Natale, 1975) or temporal aspects of the conversa-

48 tion (e.g., turn-taking, speaking rate, Beňuš, Gravano, \& Hirschberg, 2011; Street, 1984). Generally,

49 accommodation can lead to greater similarity of interlocutors' verbal patterns (e.g., Abney, Paxton,

50 Dale, \& Kello, 2014). It also plays an important role in enhancing mutual comprehension (Garrod

51 and Pickering, 2004) and cooperation (Manson et al., 2013), and in reducing the social distance

52 between interlocutors (Giles et al., 1991). In particular temporal accommodation between interlocu-

53 tors, that is mutual adaptation of temporal structure (speech rate, turn-timing), is associated with

54 positive perception (pleasantness) of a conversation (Warner et al., 1987). This type of accommoda-

55 tion evolves from very early on, in the interactions between infants (4 months) and their mothers

56 (Jaffe et al., 2001). Importantly, temporal accommodation implies the capacity of interlocutors to

57 anticipate various temporal characteristics of the partner's speech, such as the time of important

58 discourse units, ends of utterances or turns (Garrod and Pickering, 2015). Temporal predictions aid

59 interlocutors to "be on time" with their own verbal production, warranting a fluent verbal exchange

60 and sustaining the flow of interaction.

61 Anticipatory processes involved in the development of conversational skills were already found in

62 children as young as three years using eye tracking (Casillas and Frank, 2013). When watching a

63 dialogue, three-year olds shifted their gaze onto the next speaker although the current speaker had

64 not yet completed his turn. These results indirectly show that children develop early anticipatory

65 abilities inherent to the conversation. 
Temporal accommodation in children with HL

66 Hilbrink and collaborators (2015) obtained a more direct measure of these abilities by analyzing

67 dialogues between mothers and children aged from 3 to 18 months. Their analysis revealed that

68 temporal patterns of speech turns of mother-infant conversations (5 months) were similar to those

69 found in adults (i.e., with little overlap of vocalizations and a small time interval between two

70 turns). However, temporal accommodation and development of anticipatory skills in the production

71 of children at verbal age await further clarification.

72 In clinical populations, it has been shown that using rhythmic cues and training can enhance tem-

73 poral predictions in speech and thereby, may aid temporal accommodation in conversation. For ex-

74 ample, patients with dysarthria (Liss et al., 2009) display irregular vocalic and consonantal segment

75 durations altering speech rate and impacting conversational abilities. However, regular stress distri-

76 butions in the speech of an interlocutor, allowing for enhanced temporal predictions, can help

77 dysarthric patients to better accommodate to the speech of their conversational partner (Späth et al.,

78 2016). Other clinical studies have highlighted that verbal and non-verbal rhythmic training, in par-

79 ticular by combining auditory and motor stimulation, can have positive effects in patients suffering

80 from language disorders involving temporal deficits. For example, patients with non-fluent aphasia,

81 improved their speech production (i.e. articulatory quality) when speech was accompanied by a

82 regular pulse (Stahl, Kotz, Henseler, Turner, \& Geyer, 2011). Rhythmic auditory cues accompany-

83 ing speech can also enhance fluency in children and adults who stutter (Andrews et al., 1982). Chil-

84 dren with SLI and dyslexia improved their grammatical judgments after listening to a regular prime

85 (compared to an irregular prime, Przybylski et al., 2013). Children with dyslexia improved their

86 phonological and reading abilities after an active musical training and this improvement in speech

87 abilities was correlated with an improvement in their rhythmic abilities (Flaugnacco et al., 2015).

88 Children with non-verbal autism spectrum disorders increased the range and the complexity of their 
Temporal accommodation in children with HL

89 vocal productions, after using an audio-motor training (15 minutes, 5 times a week during 8 weeks)

90 as an intervention-program (Wan et al., 2011). These examples show that listening to regular

91 rhythms or training rhythmic skills helps patients who suffer from deficits in temporal processing to

92 better encode and predict the temporal structure of speech.

93 A particularly vulnerable population, with regard to the role of temporal predictions for their com-

94 municative skills, are children with hearing loss (HL). Children with cochlear implants (CIs) and/or

95 hearing aids (HAs) show high variability in spoken language achievement and difficulties in com-

96 municational skills compared to children with normal hearing (NH) (van Wieringen and Wouters,

97 2015). Pragmatic skills develop relatively late in children with hearing loss using both speech and

98 sign language, compared to children with NH (Bebko et al., 2003). Studies in children with HL de-

99 scribe inappropriate use of pragmatic features in speech turns (Most et al., 2010), greater presence

100 of silences, communication breakdowns (Tye-Murray, 2003) and excessively long speech turns

101 (Toe and Paatsch, 2013), eventually reducing the smoothness and balance of verbal exchanges.

102 These studies on pragmatic skills in children with HL clearly suggest conversational difficulties but

103 miss a link with more general temporal processing deficits. Interestingly, the few studies addressing

104 temporal prediction and processing in children with HL have been carried out in the music domain.

105 While children with CIs or HAs are able to discriminate rhythmic patterns (Innes-Brown et al.,

106 2013) children with CIs are less precise than children with NH (Stabej et al., 2012, Roy et al.,

107 2014). Electrophysiological studies using the mismatch negativity protocol (MMN) show that chil-

108 dren with CIs are less sensitive to temporal manipulation of a regular auditory structure compared

109 to NH children (Torppa et al., 2012, Petersen et al., 2015). Interestingly, it was recently shown that,

110 in a sentence repetition task, musical rhythmic priming of speech, by engaging temporal predic- 
Temporal accommodation in children with HL

111 tions, facilitated phoneme and word perception and production abilities of children with CIs and

112 HAs, (Cason et al., 2015b).

113 In light of these findings, we wanted to test whether training temporal prediction capacities in chil-

114 dren with HL via musical rhythm may impact their communication and accommodation skills in

115 production. More precisely, the aims of the present study were twofold. First, focusing on children

116 with $\mathrm{NH}$, we investigated how rhythmic predictability of turn-taking impact on temporal accommo-

117 dation skills at 5-6 years of age. Second, from a more clinical standpoint, we examined the hypothe-

118 sis that rhythmic training influences these skills in French speaking children with HL wearing coch-

119 lear implants (CIs) and/or Hearing Aid (HAs). Indeed, CI technology can deliver temporal infor-

120 mation better than spectral cues and several studies have shown a good performance of CI children

121 in rhythmical musical tasks (e.g. Innes-Brown et al., 2013, Roy et al., 2014).

122 Inspired by adaptation studies with adults (Himberg et al., 2015; Kawasaki et al., 2013; Späth et al.,

123 2016), we developed a child-friendly, novel procedure of a turn-taking paradigm (an "alternating

124 picture naming task") manipulating the difficulty of temporal anticipation required to perform the

125 task. In a first Experiment, children with NH named pictures on the screen in alternation with a vir-

126 tual partner. The temporal predictability of stress occurrences ${ }^{1}$ and thus of the turns was manipulat-

127 ed as well as the alternation rate. We measured children's temporal adaptation and prediction skills

128 by analysing the timing of the stressed syllables of words with respect to those of the virtual part-

129 ner. We hypothesized that children should adopt a regular rhythm in their own turn-production (i.e.,

130 producing their words consistently with respect to the virtual partner, Himberg et al., 2015) depend-

131 ing on the alternation rate of the partner. Moreover, we hypothesized that altering the number of

132 syllables per word and thus, lowering the predictability of time of stress occurrences should render

\footnotetext{
${ }^{1}$ We use the term "stress" throughout the manuscript in its broad sense (accent, emphasis), and not in terms of "lexical stress" which is not a feature of French, the native language of the participants in the present study.
} 
Temporal accommodation in children with HL

133 the task more difficult and deteriorate children's consistency in turn-taking. In a second Experi-

134 ment, we used the same procedure to measure the impact of rhythmic training on speech production

135 and more precisely on the temporal prediction abilities in speech in children with HL. Based on

136 previous findings with other language impaired populations (Flaugnacco et al., 2015; Przybylski et

137 al., 2013) we expected children with HL to benefit from rhythmic training in their adaptation skills, 138 especially in the less predictable context.

\section{2. Experiment 1}

141 2.1. Material and methods

\subsubsection{Participants}

143 Sixteen children with NH were recruited from a kindergarten in Marseille. They were aged from 5

144 to 6 years old (mean age $=65$ months, $S D=5$ months) and were all native-speakers of French

145 without any visual, speech, cognitive or hearing disorder. This experiment has been approved by the 146 ethics committee Sud Méditerranée I (nID RCB: 2015-A01490-49). All children's parents signed a 147 consent form.

\subsubsection{Stimuli}

150 One hundred sixty pictures from 16 taxonomic categories were selected from the French BD2I

151 database (Cannard et al., 2005). Half of them depicted monosyllabic words, half were disyllabic.

152 The selected pictures were named correctly by over $90 \%$ of a normative sample of 3 to 8 year old 153 children (monosyllabic words: mean score $=93.9 \%, S D=5.3 \%$; disyllabic words: mean score $=$ $15498.8 \%, S D=1.6 \%)$. 
Temporal accommodation in children with HL

155 The picture names were recorded in a soundproof booth and they were spoken by a female native

156 French speaker (henceforth, the virtual partner). An additional set of three words was recorded by 157 an 8-year-old boy in order to construct a model for the alternated naming procedure. The perceptual 158 centers of the syllables ( $p$-centers) were estimated at $2 / 3$ of the amplitude rise in the sound envelope 159 using a semi automatic procedure (Cummins and Port, 1998). These estimated p-centers, described

160 as being rhythmic anchor-points in speech, were used as points of reference for the adjustment of 161 the interval between the words uttered by the adult virtual partner (i.e., the Inter-Turn-Interval, ITI).

162 In disyllabic words, the p-center on the stressed syllable (i.e., the final syllable of each French 163 accentual phrase) was chosen.

164

165

166

167

168

169

170

171

172

173

174 In order to manipulate temporal predictability of the turns, pairs of pictures were created by varying

175 their syllable number ("word regularity") which modified the timing of words and their stress

176 occurrences. In a highly predictable condition, the pair was matched in the number of syllables (i.e.

177 the names of both pictures had the same number of syllables, mono- or disyllabic, respectively). In

178 the less predictable condition, the pair was mismatched (i.e. one picture name was a monosyllabic 
Temporal accommodation in children with HL

179 word and the other a disyllabic word). Four blocks were built containing each 10 pairs of pictures in

180 the matched condition (1 with monosyllabic words and 1 with disyllabic words) and 4 blocks of 10

181 pairs of pictures in the mismatched condition. Pictures in a given pair and pair order were carefully

182 controlled in order to avoid semantic, categorical or phonological priming between words of the

183 same pair or between two successive pairs. In a given block, the alternation rate was determined by

184 the ITI of the virtual partner. It was either a fast rate (i.e., ITI of $2600 \mathrm{~ms}$ between the p-centers of

185 two successive words of the virtual partner, see Figure 1) or slow (i.e., ITI of 3200 ms). This

186 resulted in a 2 by 2 factorial design manipulating word regularity (matched or mismatched) and

187 alternation rate (slow or fast) across blocks. The order of block presentation was counterbalanced

188 across participants.

189 Each block started with 3 pairs of pictures that were named by the adult virtual partner in perfectly

190 timed alternation with the voice of the 8-year-old boy in order to establish the rate of alternation in

191 an implicit manner. Next,10 pairs of pictures were presented successively at the bottom of the

192 screen in a scrolling manner and the child was instructed to name the pictures continuing the

193 alternation. Children naturally tended to name the images in between the two successive

194 pronunciations of the virtual partner. No information concerning when to pronounce the name of

195 the image nor on word regularity and alternation rate were given to the child. A block was not

196 interrupted even if the child misnamed or did not name a picture. The child was praised and

197 encouraged to continue after completion of each block.

198 Stimuli delivery was programmed using the software Presentation (Neurobehavioral Systems 18.0).

199 Pictures were displayed on a laptop screen (DELL Latitude E5530, screen resolution 1366 X 768 ).

200 The volume for the voice of the virtual partner, delivered by a CREATIVE sound card (X-Fi 5.1)

201 and two Sony loudspeakers (SRS A205), was adjusted for each child at a comfortable level. The 
Temporal accommodation in children with HL

naming performance of the participant was recorded with a head-mounted microphone (Sennheiser HSP) linked to a ZOOM H4n numeric recorder at a $44 \mathrm{~Hz}$ sampling rate.

\subsubsection{Analysis}

207 Inter-word-intervals between the child and the virtual partner and the relative timing of children's

208 words to the words of the virtual partner were chosen as measures to evaluate temporal 209 accommodation (Himberg et al., 2015). In order to determine the Inter-Word-Interval (IWI)

210 between the virtual partner and the child, we estimated the distance of the p-centers of the stressed

211 syllables within each pair of words as pronounced by the virtual partner and the child (see Figure

212 1). The IWI was chosen in order to evaluate children's accommodation to the different alternation

213 rates (fast and slow). Intervals were excluded from analysis if the child made a hesitation (e.g.,

214 euh), erroneously placed an article before naming the object or spoke simultaneously with the

215 virtual partner. This resulted in rejecting approximately $10 \%$ of word-intervals. Then, we analyzed

216 the timing of children's stressed syllables in relation to those of the virtual partner. Because of the

217 highly predictable rhythm of alternation, it was expected that children would anticipate the time of

218 the words (their stressed syllable) of the virtual partner and, consequently, produce their own words

219 (stressed syllables) in a stable temporal relationship to the virtual partner. This anticipatory

220 behavior was confirmed in a pilot study on three children. A preferred and particularly stable

221 temporal relation in rhythmic tasks requiring an action in response to a regular rhythmic auditory

222 structure is the anti-phase (i.e., the mid-point between two periodically recurring auditory events;

223 Repp, 2005; Volman \& Geuze, 2000). Thus, the timing of the child's stressed syllables was

224 analyzed, as a default, with respect to this 'expected' moment in time representative of a temporally 
Temporal accommodation in children with HL

225 perfectly regular alternation (i.e., half of the ITI of the virtual partner, notably, 1300 and $1600 \mathrm{~ms}$

226 after the p-center of the virtual partner for the slow and fast condition, respectively). The analysis is

227 best performed using circular statistics (CircStat Toolbox, Berens, 2009). Thereby, each child's

228 response is expressed as a vector in a circular space with respect to the expected moment in time

229 (see Supplemental material, appendix A for more details). In this space, the angle represents the

230 asynchrony (i.e., the distance between the child's production and the expected moment in time) and

231 the length of the vector represents the consistency (i.e., the variability of the child's asynchrony

232 across trials). Consistency is particularly useful to estimate the child's temporal accommodation

233 skills as more consistent (less variable) performances are indicative of a better temporal

234 representation of the reciprocal turn-structure. Previous to any statistical inference, the Rayleigh test

235 was used to verify that performances were not randomly distributed in the circular space (that is not

236 uniformly distributed). None of the children assessed in this study had a uniform distribution,

237 showing that they had they had indeed produced the stressed syllables of the words in a consistent

238 and anticipatory fashion with respect to the virtual partner's stresses.

239

240 2.2. $\underline{\text { Results }}$

241 Three 2 × 2 repeated-measures analysis of variance (RM-ANOVA) with the factors Alternation rate

242 (fast versus slow) and Word regularity (matched versus mismatched stress occurrences) and the

243 mean Inter-Word-Interval and consistency as the dependent variables were performed.

244 IWI analyses revealed a main effect of alternation rate: children with NH showed longer intervals in

245 the slow than in the fast alternation condition $(F(1,15)=24.99, p<.001$; see Fig. 2A). Consistency

246 analyses showed that children were lass variable (i.e. more consistent) in the fast than in the slow

247 condition (main effect of rate: $F(1,15)=6.85, p=.019$; Fig. 2C). In addition, they showed a tendency 
Temporal accommodation in children with HL

248 to be less variable when the pairs of words had a regular instead of an irregular stress pattern (main

249 effect of regularity: $F(1,15)=4.09, p=.061$; Fig. 2 C).

\subsection{Discussion}

253 These findings show that children with $\mathrm{NH}$, as young as 5 years of age, are sensitive to temporal

254 variations (alternation rate and the timing of words' stressed syllables) in the turns of their 255 interlocutor. As a matter of fact, results on IWI revealed that children are able to adapt their naming 256 performance to the rate of the virtual partner. This temporal adaptation (fast vs. slow: $\Delta=157 \mathrm{~ms}$ ) is

257 an important finding as it demonstrates that children were not simply reacting to the visual stimulus

258 they had to name, but they were indeed taking into account changes in the temporal pace of 259 alternation. Hence, our task, as intended, was apt to measure children's temporal attunement 260 abilities in a pseudo-interactive setting.

261 We also observed a trend for facilitated adaptation when sequences of words were more predictable 262 in their timing properties, that is in the matched condition, wherein the child and the virtual words 263 partner had the same number of syllables (i.e. the same stress patterns). Indeed, the mismatched 264 condition seemed to perturb the dynamics of the interaction by slightly degrading the phase-locking 265 between the child and the virtual partner. Note that a disyllabic word with a final stress needs to be 266 initiated earlier than a monosyllabic word. Because the alternating naming task requires the child to 267 anticipate the moment of her/his turn in relation to the naming interval of the virtual partner, chang268 ing the number of syllables of words may degrade anticipatory strategies or put higher demands on 269 the anticipatory mechanism in general. 
Temporal accommodation in children with HL

\section{3. Experiment 2}

276 In Experiment 2, we used the same paradigm in order to investigate the capacities of temporal ac277 commodation in children with CIs or HAs following a speech therapy session and a rhythmic musi278 cal training.

279 We hypothesized that children with HL would benefit more strongly from a rhythmic training ses-

280 sion in their adaptation skills, compared to a speech therapy session. This effect should be more 281 evident in mismatched condition, that is when the temporal predictability of word exchanges was 282 more difficult.

\section{$\underline{\text { 3.1. Material and methods }}$}

286 Fifteen children with HL aged from 5 to 9 years (mean age $=89$ months, $S D=17$ months) were

287 recruited from the Centre d'Action Médicale Sociale Précoce in Marseille (children aged from 5 to

2886 years) and the Service de Soutien à l'Education Familiale et à la Scolarisation in Marseille

289 (children aged 6-9 years). Some of the children wore a unilateral CI, others wore bilateral CIs, some 290 wore a cochlear implant (CI) in conjunction with a conventional hearing aid (HA) on the

291 contralateral ear and others HAs. Even if CI delivers an electric signal to the auditory nerve versus

292 an amplified acoustic signal for HA, users of both devices may benefit from a rhythmic training.

293 The children suffered from different degrees of hearing loss (from profound to moderate, see Table 
Temporal accommodation in children with HL

2941 in the Supplemental material, appendix B ,where also the aided thresholds and other details of the

295 subjects are given). They were all native French speakers, educated in oral communication,

296 attended mainstream primary school, and were free from known visual, speech or other cognitive

297 impairment. Data of two of the 15 participants had to be excluded as they were unable to perform

298 the task. This experiment has been approved by the ethics committee ( $\mathrm{n}^{\circ}$ ID RCB: 2015-A01490-49,

299 Sud Méditerranée I). All children's parents signed a consent form.

300 3.1.2. Stimuli, procedure and analyses

301 The stimuli, the task and data analyses were the same as in Experiment 1. However, participants of

302 Experiment 2 performed the naming task twice. Once, the measure was taken 30 minutes after a

303 training session focusing on musical rhythmic stimulation which was meant to enhance temporal

304 prediction skills in children. The training included several rhythmic audio-motor games (for more

305 details, see Supplemental material, appendix C). As a comparison, a 'baseline' measure was taken

30630 minutes after a speech therapy session that focused on verbal stimulation in a classical speech

307 therapy setting. Indeed, in order to avoid a general effect of training on arousal or mood that would

308 render results difficult to interpret (Thompson et al., 2001) we opted for this 'baseline' measure

309 instead of a non-stimulation baseline. Both types of stimulations lasted 30 minutes and used a

310 playful and joyful setup. The 2 sessions were separated by one week on average and the order of

311 stimulation was counterbalanced across children.

312

313 3.2. $\underline{\text { Results }}$

314 Data were entered into a three 2x2x2 RM ANOVA with Alternation rate (fast versus slow), Word 315 regularity (matched versus mismatched stress occurrences), and Training type (speech therapy 
Temporal accommodation in children with HL

316 versus rhythmic training) as within subject factors and IWI (Inter-Word-Interval) and consistency

317 (i.e. regularity) dependent variables.

318 Analyses on the IWI revealed a main effect of alternation rate on mean IWI: children with HL

319 produced longer IWI in the slow than in the fast condition $(F(1,12)=10.95, p=.006)$. Consistency

320 analyses showed that children with HL were generally more consistent in the fast than in the slow

321 condition (consistency: $F(1,12)=8.72, p=.012$.

322 As Fig. 3 shows, we found no main effect of regularity on consistency $(p=.414)$ but an interaction

323 effect of training type and word regularity $(F(1,12)=7.99, p=.015)$.

\subsection{Discussion}

326 Children with HL were generally able to adapt their rate of production to the rate of the virtual 327 partner and were generally more consistent in the fast than in the slow condition as NH children.

328 However, children with HL showed no differences in consistency when the pairs of words had a

329 regular or an irregular number of syllables after the speech therapy session. However, and most

330 importantly, after musical training, children with HL became more consistent in their alternation

331 pattern in the matching than in the non-matching condition. This suggests that they were more

332 sensitive to the temporal regularity of the speech exchanges after the rhythmic training. Note that

333 (the younger) children with NH in Experiment 1 responded in a similar way (i.e., matched >

334 mismatched stress occurrences), but without musical training.

\section{4. General discussion}

337 In sum, the present study showed that children with $\mathrm{NH}$ as young as 6 years of age were able to 338 temporally anticipate and accommodate to the turn-structure of a virtual partner in a non-random 
Temporal accommodation in children with HL

339 way. Results showed that they were sensitive to the temporal predictability of turns and that they

340 temporally adapted their speech production better to faster than slow turn rhythms. Children with

341 HL, after having received speech or musical training also managed to perform the task and adapted

342 better to fast than to slow turn rhythms. However, in contrast to the younger NH children, they did

343 not benefit from more predictable turn-structure, unless they received musical rhythmic training.

344 Most importantly, this is, to our knowledge, the first study to provide evidence that a rhythmic

345 training of only 30 minutes may enhance the capacity of children with HL to accommodate to a

346 temporally predictable flow of turn exchanges in an interactional setting. This effect points to po-

347 tential benefits of rhythmic training on audio-motor anticipatory skills in children with HL. Rhyth-

348 mic training may also have improved the extraction of regularities along the series of speech ex-

349 changes. By grouping discrete events (e.g. words) in a more hierarchical structure, children may

350 have better perceived the metrical structure of the turns (i.e., the recurrence of stresses / syllables),

351 allowing for a more precise temporal prediction of their own and their partner's turns.

352 Previous work has shown that musical training in adults enhances connectivity within a dorsal net-

353 work comprising the auditory and the motor cortex (Chen, Penhune, \& Zatorre, 2008). This net-

354 work plays an important role in auditory predictions (Schubotz, 2007) and attentional dynamics

355 (Schroeder et al., 2010). There is a possibility that rhythmic training in children with HL may

356 stimulate the sensori-motor dorsal pathway and thereby improve children's abilities to adapt to a

357 regular temporal structure (Vuust and Witek, 2014) or more generally improve their ability to per-

358 ceive and understand rhythmical and metrical structure (Cason et al., 2015a).These different possi-

359 bilities should be investigated in further detail in future studies.

360 Overall, our results are in line with previous studies showing that sensori-motor rhythmic training

361 enhances auditory processing in different forms of language impairment at cortical and subcortical 
Temporal accommodation in children with HL

362 levels (Flaugnacco et al., 2015; Fujii \& Wan, 2014; Tierney \& Kraus, 2013). Concerning the sen-

363 sorimotor rhythmic capacities of adults with CIs, their lack of synchronization capacity on a com-

364 plex musical stimulus (Phillips-Silver et al., 2015) could be indicative of the fact that meter (i.e., the

365 regular recurrence of a beat or accents) perception and / or production is underdeveloped in hearing

366 loss. Our data in children with HL seem to confirm these previous findings in adults. Interestingly,

367 similarly to adults listening to complex auditory stimuli, children with HL, even after a speech ther-

368 apy session, do not take advantage of the temporal regularity of stress occurrences in our task, while

369 children with NH do. As metrical perception is shaped by auditory experience (Hannon and Trehub,

370 2005), musical practice may refine this type of perception in both music (Geiser et al., 2010), and

371 speech (Marie et al., 2011). Developing the perception of meter in children with hearing loss may

372 thus improve their general temporal anticipatory skills and thus enhance their ability to adapt in

373 simple as well as in more complex speech interaction contexts. Moreover, the enjoyment related to

374 music training and more specifically the fact of being actively involved in synchronizing with

375 somebody during 30 minutes, may also have enhanced children's engagement in the interactional

376 task and improved their consistency after rhythmic training (Kirschner and Tomasello, 2009).

\section{5. Conclusion, limitations and perspectives}

379 This study presented a new task which allows for examining temporal adaptation in children at 380 verbal age in an interactive situation with a virtual partner. Results showed the relevance of 381 rhythmic musical training to improve temporal skills in children with HL. The future development 382 of this task, including the use of a more adaptive virtual agent and more complex utterances going 383 beyond the use of single words, will help us to better understand on which level of conversational 384 coordination rhythmic musical training can impact the most (Phillips-Silver and Keller, 2012). As 
Temporal accommodation in children with HL

385 our study was confined to a very periodic structure of turns, future investigations may extend to other time-sensitive phenomena in less constrained speech interaction, such as speech convergence (Pardo, 2006) which could benefit interlocutors by mutually reducing cognitive load and facilitating prosocial behavior (Manson et al., 2013).

As the musical training session was conceived to focus on rhythmic skills and entrainment, it may have enhanced not only predictive skills, but also auditory processing in general. This may, in turn, have enhanced encoding of acoustic cues such as pitch, intensity and duration, that characterize stress patterns (Torppa et al., 2014). Overall, this work supports the idea that musical stimulation in general and sensori-motor rhythmic training in particular can be integrated in speech and language rehabilitation of children with HL. Further studies should also address a direct comparison of children with $\mathrm{NH}$ and HL, which was beyond the scope of this work. Our results are also encouraging from a clinical perspective. Speech therapists with an appropriate focused musical training could develop simple but recurrent rhythmic activities with their patients with HL. Moreover, these rhythmic activities could also be carried out in a group, which was not feasible in the context of this protocol that required individual testing immediately after the training session.

\section{Acknowledgements}

This work was supported by the Brain and Language Research Institute (BLRI, ANR-11-LABEX0036 to C.H., S.F. and D.S.), the European Union Seventh Framework Program (FP7-PEOPLE2012-IEF, $\mathrm{n}^{\circ} 327586$ to S.F.), and the LMUexcellent program within the framework of the German excellence initiative (to S.F.). 
Temporal accommodation in children with HL

410 References

411 Abney, D.H., Paxton, A., Dale, R., Kello, C.T., 2014. Complexity matching in dyadic conversation.

412 J. Exp. Psychol. Gen. 143, 2304-2315. doi:10.1037/xge0000021

413 Andrews, G., Howie, P.M., Dozsa, M., Guitar, B.E., 1982. Stuttering. J. Speech Lang. Hear. Res. $414 \quad 25,208$. doi:10.1044/jshr.2502.208

415 Bebko, J.M., Calderon, R., Treder, R., 2003. The Language Proficiency Profile-2: Assessment of 416 the Global Communication Skills of Deaf Children Across Languages and Modalities of Expression. J. Deaf Stud. Deaf Educ. 8, 438-451. doi:10.1093/deafed/eng034

418 Beňuš, Š., Gravano, A., Hirschberg, J., 2011. Pragmatic aspects of temporal accommodation in turn-taking. J. Pragmat. 43, 3001-3027. doi:10.1016/j.pragma.2011.05.011

Berens, P., 2009. CircStat: A MATLAB toolbox for circular statistics. J. Stat. Softw. 31, 1-21. doi:10.1002/wics.10

424 Cannard, C., Blaye, A., Scheuner, N., Bonthoux, F., 2005. Picture naming in 3- to 8-year-old 425 French children: methodological considerations for name agreement. Behav. Res. Methods 37, 417-425. doi:10.3758/BF03192710

427 Casillas, M., Frank, M.C., 2013. The development of predictive processes in children's discourse 428 understanding. Proc. 35th Annu. Meet. Cogn. Sci. Soc. 299-304.

429 Cason, N., Astésano, C., Schön, D., 2015a. Bridging music and speech rhythm: rhythmic priming 430 and audio-motor training affect speech perception. Acta Psychol. (Amst). 155, 43-50. 
Temporal accommodation in children with HL

doi:10.1016/j.actpsy.2014.12.002

432 Cason, N., Hidalgo, C., Isoard, F., Roman, S., Schön, D., 2015b. Rhythmic priming enhances

433 speech production abilities: evidence from prelingually deaf children. Neuropsychology 29,

$434 \quad 102-7$.

435 Chen, J.L., Penhune, V.B., Zatorre, R.J., 2008. Listening to musical rhythms recruits motor regions

436 of the brain. Cereb. cortex 18, 2844-2854. doi:10.1093/cercor/bhn042

437 Cummins, F., Port, R., 1998. Rhythmic constraints on stress timing in English. J. Phon. 26, 145-

438 171. doi:10.1006/jpho.1998.0070

439 Dalla Bella, S., Sowiński, J., 2015. Uncovering Beat Deafness: Detecting Rhythm Disorders with

$440 \quad$ Synchronized Finger Tapping and Perceptual Timing Tasks. J. Vis. Exp. 1-11.

$441 \quad$ doi: $10.3791 / 51761$

442 Flaugnacco, E., Lopez, L., Terribili, C., Montico, M., Zoia, S., Schön, D., 2015. Music Training

443 Increases Phonological Awareness and Reading Skills in Developmental Dyslexia: A

444 Randomized Control Trial. PLoS One 10, e0138715. doi:10.1371/journal.pone.0138715

445 Fujii, S., Wan, C.Y., 2014. The Role of Rhythm in Speech and Language Rehabilitation: The SEP

446 Hypothesis. Front. Hum. Neurosci. 8, 1-15. doi:10.3389/fnhum.2014.00777

447 Garrod, S., Anderson, A., 1987. Saying what you mean in dialogue: A study in conceptual and

448 semantic co-ordination. Cognition 27, 181-218. doi:10.1016/0010-0277(87)90018-7

449 Garrod, S., Pickering, M.J., 2015. The use of content and timing to predict turn transitions. Front.

$450 \quad$ Psychol. 6, 1-12. doi:10.3389/fpsyg.2015.00751

451 Garrod, S., Pickering, M.J., 2004. Why is conversation so easy? Trends Cogn. Sci. 8, 8-11.

452 doi:10.1016/j.tics.2003.10.016 
Temporal accommodation in children with HL

453 Geiser, E., Sandmann, P., Jäncke, L., Meyer, M., 2010. Refinement of metre perception - training 454 increases hierarchical metre processing. Eur. J. Neurosci. 32, 1979-1985. doi:10.1111/j.14609568.2010.07462.x

Giles, H., Coupland, N., Coupland, J., 1991. Accomodation theory: Communication, context, and consequence, in: Giles, H., Coupland, J., Coupland, N. (Eds.), Contexts of Accomodation:

Hannon, E.E., Trehub, S.E., 2005. Metrical categories in infancy and adulthood. Psychol. Sci. 16, 48-55. doi:10.1111/j.0956-7976.2005.00779.x

Hilbrink, E.E., Gattis, M., Levinson, S.C., 2015. Early developmental changes in the timing of turn-

464 Himberg, T., Hirvenkari, L., Mandel, A., Hari, R., 2015. Word-by-word entrainment of speech rhythm during joint story building. Front. Psychol. 6, 1-6. doi:10.3389/fpsyg.2015.00797

Innes-Brown, H., Marozeau, J.P., Storey, C.M., Blamey, P.J., 2013. Tone, rhythm, and timbre taking: a longitudinal study of mother-infant interaction. Front. Psychol. 6, 1-12. doi:10.3389/fpsyg.2015.01492

Jaffe, J., Beebe, B., Feldstein, S., Crown, C.L., Jasnow, M.D., 2001. Rhythms of dialogue in infancy: coordinated timing in development. Monogr. Soc. Res. Child Dev. 66, 1-132.

472 Kawasaki, M., Yamada, Y., Ushiku, Y., Miyauchi, E., Yamaguchi, Y., 2013. Inter-brain synchronization during coordination of speech rhythm in human-to-human social interaction. doi: $10.2307 / 3181589$ Sci. Rep. 3, 1-8. doi:10.1038/srep01692

Kirschner, S., Tomasello, M., 2009. Joint drumming: Social context facilitates synchronization in 
Temporal accommodation in children with HL

preschool children. J. Exp. Child Psychol. 102, 299-314. doi:10.1016/j.jecp.2008.07.005

Liss, J.M., White, L., Mattys, S.L., Lansford, K., Lotto, A.J., Spitzer, S.M., Caviness, J.N., 2009. Quantifying speech rhythm abnormalities in the dysarthrias. J. Speech. Lang. Hear. Res. 52,

Manson, J.H., Bryant, G. a., Gervais, M.M., Kline, M. a., 2013. Convergence of speech rate in conversation predicts cooperation. Evol. Hum. Behav. 34, 419-426. 1334-52. doi:10.1044/1092-4388(2009/08-0208)

Marie, C., Magne, C., Besson, M., 2011. Musicians and the metric structure of words. J. Cogn. Neurosci. 23, 294-305. doi:10.1162/jocn.2010.21413

Most, T., Shina-August, E., Meilijson, S., 2010. Pragmatic abilities of children with hearing loss using cochlear implants or hearing AIDS compared to hearing children. J. Deaf Stud. Deaf

Natale, M., 1975. Convergence of Mean Vocal Intensity in Dyadic Communication as a Function of

Pardo, J.S., 2006. On phonetic convergence during conversational interaction. J. Acoust. Soc. Am. 119, 2382. doi:10.1121/1.2178720

Petersen, B., Weed, E., Sandmann, P., Brattico, E., Hansen, M., SÃ rrensen, S.D., Vuust, P., 2015. Brain Responses to Musical Feature Changes in Adolescent Cochlear Implant Users. Front. implant users move in time to the beat of drum music. Hear. Res. 321, 25-34. 
Temporal accommodation in children with HL

Przybylski, L., Bedoin, N., Krifi-Papoz, S., Herbillon, V., Roch, D., Léculier, L., Kotz, S. a, with developmental language disorders. Neuropsychology 27, 121-31. doi:10.1037/a0031277

Repp, B.H., 2005. Sensorimotor synchronization: A review of the tapping literature. Psychon. Bull. Rev. 12, 969-992. doi:10.3758/BF03206433

Roy, A.T., Scattergood-Keepper, L., Carver, C., Jiradejvong, P., Butler, C., Limb, C.J., 2014.

Schroeder, C.E., Wilson, D.A., Radman, T., Scharfman, H., Lakatos, P., 2010. Dynamics of Active Sensing and perceptual selection. Curr. Opin. Neurobiol. 20, 172-176.

Schubotz, R.I., 2007. Prediction of external events with our motor system: towards a new

$$
\text { framework. Trends Cogn. Sci. 11, 211-218. doi:10.1016/j.tics.2007.02.006 }
$$

Skodda, S., Flasskamp, A., Schlegel, U., 2010. Instability of syllable repetition as a model for impaired motor processing: Is Parkinson's disease a "rhythm disorder”? J. Neural Transm. 117, 605-612. doi:10.1007/s00702-010-0390-y

Späth, M., Aichert, I., Ceballos-Baumann, A.O., Wagner-Sonntag, E., Miller, N., Ziegler, W., 2016. Entraining with another person's speech rhythm: Evidence from healthy speakers and individuals with Parkinson's disease. Clin. Linguist. Phon. 9206, 1-18. doi:10.3109/02699206.2015.1115129 abilities of prelingually deaf children with cochlear implants. Int. J. Pediatr. Otorhinolaryngol. 
Temporal accommodation in children with HL

523 Stahl, B., Kotz, S.A., Henseler, I., Turner, R., Geyer, S., 2011. Rhythm in disguise: Why singing 524 may not hold the key to recovery from aphasia. Brain 134, 3083-3093. doi:10.1093/brain/awr240

Street, R., 1984. Speech convergence and speech evaluation in fact-finding interviews. Hum. Commun. Res. 11, 139-169. doi:10.1111/j.1468-2958.1984.tb00043.x

528 Thompson, W.F., Schellenberg, E.G., Husain, G., 2001. Arousal, Mood, and The Mozart Effect. Psychol. Sci. 12, 248-251. doi:10.1111/1467-9280.00345

530 Tierney, A., Kraus, N., 2013. Music training for the development of reading skills., 1st ed, Progress in brain research. Elsevier B.V. doi:10.1016/B978-0-444-63327-9.00008-4

Toe, D.M., Paatsch, L.E., 2013. The conversational skills of school-aged children with cochlear implants. Cochlear Implants Int. 14, 67-79. doi:10.1179/1754762812Y.0000000002

534 Torppa, R., Faulkner, A., Huotilainen, M., Järvikivi, J., Lipsanen, J., Laasonen, M., Vainio, M., 535 2014. The perception of prosody and associated auditory cues in early-implanted children: the 536 role of auditory working memory and musical activities. Int. J. Audiol. 53, 182-91. doi:10.3109/14992027.2013.872302

538 Torppa, R., Salo, E., Makkonen, T., Loimo, H., Pykäläinen, J., Lipsanen, J., Faulkner, A., Huotilainen, M., 2012. Cortical processing of musical sounds in children with Cochlear Implants. Clin. Neurophysiol. 123, 1966-1979. doi:10.1016/j.clinph.2012.03.008 82S-89S. doi:10.1097/01.AUD.0000051691.33869.EC

544 implanted at a young age with respect to their auditory, linguistic and cognitive skills? Hear. 
Temporal accommodation in children with HL

Res. 322, 171-179. doi:10.1016/j.heares.2014.09.002

546 Volman, M.J., Geuze, R.H., 2000. Temporal stability of rhythmic tapping "on" and "off the beat": a

547 developmental study. Psychol. Res. 63, 62-9. doi:10.1007/PL00008168

548 Vuust, P., Witek, M. a. G., 2014. Rhythmic complexity and predictive coding: a novel approach to 549 modeling rhythm and meter perception in music. Front. Psychol. 5, 1-14.

$550 \quad$ doi:10.3389/fpsyg.2014.01111

551 Wan, C.Y., Bazen, L., Baars, R., Libenson, A., Zipse, L., Zuk, J., Norton, A., Schlaug, G., 2011.

552 Auditory-motor mapping training as an intervention to facilitate speech output in non-verbal

553 children with autism: A proof of concept study. PLoS One 6, 1-7.

554 doi:10.1371/journal.pone.0025505

555 Warner, R.M., Malloy, D., Schneider, K., Knoth, R., Wilder, B., 1987. Rhythmic organization of 556 social interaction and observer ratings of positive affect and involvement. J. Nonverbal Behav.

$557 \quad$ 11, 57-74. doi:10.1007/BF00990958

558 Zatorre, R.J., Chen, J.L., Penhune, V.B., 2007. When the brain plays music: auditory-motor

559 interactions in music perception and production. Nat. Rev. Neurosci. 8, 547-58.

560 doi: $10.1038 / \mathrm{nrn} 2152$

561

$562 \quad$ Figure legends

563 Figure 1. Representation of the time course of 2 trials of a fast alternation block. The thick rectangle

564 indicates the current pair of pictures. The virtual partner names the pictures on the left (trial 1: knife 565 fr.couteau), while the child has to name the pictures on the right (trial 1: baby, fr.bébé). Corre566 sponding representation of the speech signal. The ticks indicate the location of the virtual partner $\mathrm{p}$ 567 centers (p 0, p 2600) and, for the child, the expected location of the child p-centers (p 1300, p 
Temporal accommodation in children with HL

568 3900). The actual child p-centers, in this example, are indicated by an arrow and are slightly ahead

569 the expected moment.

570

571 Figure 2. Panel A: Children's (NH) temporal accommodation with a virtual partner. Error bars

572 represent the standard error of the mean. Left: mean IWIs in seconds between the p-centers of the

573 stressed syllables of the child and those of the virtual partner in the Slow and Fast conditions. Right:

574 Consistency (vector length) of the child's word productions as a function of word regularity. Panel

575 B: Temporal accommodation with a virtual partner in children with HL. Left: Mean IWIs in

576 seconds between the p-centers of the child and those of the virtual partner in the Slow and Fast

577 conditions. Right: Consistency (vector length) of the child's word production as a function of word

578 regularity in both training conditions. P-values are results of post-hoc tests.

579

580

581

582

583

584

585

586

587

\section{$\underline{\text { Supplemental material }}$}

Appendix A: Measuring consistency with circular statistics.

Appendix B: Details on the children with hearing loss

Appendix C: Rhythmic musical training 